\title{
Digital Technology in the Analogue Experience
}

\author{
Christopher Morgan \\ Tasmanian Secondary Polytechnic/Academy, Australia
}

\begin{abstract}
Intuitive and creative development begins as a process in infancy and is seen by many childhood theorists as being essential to the development of literacy and numeracy skills as the child progresses through education. This development is also essential to the development of the individual and fundamental to the building of a personal relationship with the environment. The environment that humans inhabit and that children live within to gain the experiences required to develop and grow, is revealed through analogue wavelengths to the human senses. The environmental and analogue phenomenon of colour, tone, line, texture, etc., forms the building blocks of experience and the wavelengths have a direct bearing on the human sensual receptors. It is important that the sources of stimuli for the infant and child are analogue and that the evolutionary notion of sensual awareness remains pre eminent as the source for an understanding of life. Analogue waves bring colour, shape, texture, smell etc to the growing child, hopefully in a caring environment that assists but doesn't control the child's experiences. It is this naïve and selfgoverning experience that needs to be preserved through infancy and early education. A purely digital and technological learning experience can be highly motivating and can be seen as a tool for progress, but it should be used as an adjunct, not a replacement, for the analogue experience. Purely digital sources are contrived and represent human adult construction. A balanced combination of the two, analogue and digital, creates digital opportunity.
\end{abstract}

\section{Introduction}

An infant has great potential for awareness of the world in which it lives. Each adult person has a capacity to contribute to a society as a creative and inventive individual. The response of the infant or child to analogue stimuli becomes a contribution to its world but this natural urge has a tendency to wane in our society as the child grows. Paying lip service to natural and instinctive processes and contributions that emanate from each infant and labeling the efforts of these individuals to develop and contribute as cute, quaint or insignificant represents a potential breaking up of the individual mind and body in society. This results in the embarking of the child on a pathway to an eventual station in life as part of a technological and industrial society. Keepers of our society perceive a future for infants and children that is actually impossible to perceive, as the compatibility of current educational practices with technological advancement is questionable.

The capacity for a person to operate as a literate being significantly emerges from the experiences and opportunities available during infancy. One of the outward manifestations of these experiences can be seen in the seemingly meaningless scribbles, drawn strokes and ill formed circles that emerge during childhood. These symbolic scribbles and notations are an outward sign of a growing inner awareness. As the scribbles form and are controlled by each child, shapes emerge from the scribbles and become isolated and drawn as individual shapes. Patterns and shapes both negative and positive become representations of a perceived world. The shapes that are perceived in the scribbles come to the infant or child through personal experience as unique shapes formed by the hand that began to act out the scribble in the first place. The scribbles and shapes bear similarities to the efforts of all other scribblers and shape makers, that is, those who have not been bound by societal strictures. As all human desire is to progress through these early and infant stages, then we have indeed a global connection involving both the individual and society.

Human invention, creativity and interaction have a foundation based on this developmental phenomenon. The building blocks for the development of human imagination exist within the perception we have of the environment around us and our freedom and intuition to 
explore and learn from this environment is commensurate with our personal requirements for this process to happen.

Digital technology creates a contrast to the analogue world experience in its most basic form. Human experiences, being from an analogue environment, essentially provide different experiences from what is revealed on a digital screen and in virtual worlds. In a digital context, aspects of another person's analogue experience are digitized or converted into number sequences to approximate aspects of the environment, which humans commonly share. Only sensual experiences that lend themselves to this practice are encountered. Sight and sound, then, become the dominant senses and avenues to receive information, one supporting the other in its presence or in its absence. An emphasis on the senses of sight and sound has historically been the case with formal education in the development of language and the arts in modern western culture, including the invention of recording technologies. Analogue recording technologies harness natural waveforms and reproduced them via valves, transistors, chemical reaction or fields of interpolation. Digital technology has replaced the natural waveform, the essence of the analogue, with a devised number system. Digital technology offers undoubted clarity. Outlining and isolating in the representation of natural forms has been the result of this digital and technological revolution. There is an obvious simplification in the digital representation that mimics the analogue but, within this, a finite edging is created. Human intervention, implicit in this technology, represents the possibility for greater human control over the environmental experiences of infants, children and in fact all people. A screen reality is preferred over the seemingly random reality of the natural environment if the techniques used to bring screen experiences preempt and take the place of the natural order and process of infant and childhood development. Learning by sensual association in this context becomes an artificial concoction exercising two senses only as mentioned and the development of human gestural, verbal, visual and written communication develops in command mode as the techniques of digital media demand simplification around a viewing space that has an aspect ratio of 9:16. This phenomenon is fine if the child has another reality that is closer to its natural processes with which to compare this digital and electronic representation.
Therefore, much care needs to be taken in the creation and dissemination of opportunity for a child to engage in literacy, numeracy and learning programs. A purely digitally based program leaves infants and children initially confused before the "Wow" factor sets in and takes away both natural innocence and curiosity. In other words, a purely digitally created learning environment will motivate most students. With the burden of an analogue experience taken away, the unadulterated digital experience leaves a result that looks fine as an outcome. What is gained, however, as far as the development of the imagination (and therefore, potential) for the analogue based human, is often questionable. The initial raw state of confusion, which is sorted or worked out by natural development quickly, becomes sophisticated and short circuited as digital commerce reaches further and further with its tentacles into the pool of humanity. Electronic and digital systems are logical avenues for advertising and wealth creation as there is the ability to almost simultaneously reach a global audience with the same simple message. Areas once thought to be off limits or protected by a naturally occurring family unit are entered in an attempt to commercialize the soul and spirit of people and societies for the gain of monetary wealth and power. Techniques for infiltration are developed and used by this sophisticated system which works so well that its produce for children is unquestioningly accepted as an answer to the hectic demands of bringing up a child in a fast paced commercial world. Increasingly these products are digitally based or created as recognizable objects that have no smell, texture and possibly an artificial or toxic taste. Are these simulated experiences then to be transferred through association to a world that exists for children moving forward in development? What happens when chronological age takes them into a time of acquiring their own children? Do these tastes and textures transfer into a digitally constructed world that children are increasingly encouraged to join as a means of escape from an analogue based life because they just don't understand the natural and built worlds into which they were biologically born? Is shortcircuiting of natural infant and childhood experiences in part creating collective groups of like conditioned young people? Education systems which may use regimentation as a means of coping with large groups of students need to take heed of the current actions of collective groups of confused youth whom they have directed for the latter fifteen of their eighteen 
years of life. Parenting groups need to be brought up to speed, somehow, on the isolating techniques used by advertisers to gain power over the purchasing and persuasive abilities of children.

Digital conditioning comes at a cost. Analogue based experiences teach consequences. In a digitally constructed environment no physical harm can come to any child who engages in digital risk. There is therefore little connection between the analogue and the digital environments in this context, even though digital experiences currently arrive to the sensual capacities of sight and sound through analogue means. Conditioning is a process of negating thought in regard to making connections through critical thinking. Actions that come from the mimicking of playmaking from digital entertainment have no perceived consequences for the perpetrator when carried out in a nondigital environment, as there were no consequences in the digital environment where childhood and infant development took place. Much activity of so-called digital natives is based around digital manipulation for the purposes of entertainment. Entertainment replaces play. Digital entertainment requires little critical thought or analysis as its creators take this requirement away. Rather than technology being used for creative and inventive activity, it is too often used to create an external state where commercially based entertainment products become the reason for the non productive gathering together of people in a digital solidarity. The perception of action and fun is that a "real life" re enactment of a digital adventure is as sanitized as the original entertainment experience. Digital re enactments become sanitized through "realistic" makeup and costuming and special effects, as the representation becomes the "real" for the complete and competitive digital native. The arbitrators of the success of this process are usually those who have, themselves, only ever experienced this kind of reality. Their expertise and opinion is sometimes broadcast as a representation of an ideal state of mind.

There is a movement that does not support the introduction of screen-based technology to infants and children until a time in their development when appropriate decision making processes have been formed within the young persons themselves. Often the most academic and most skilled young person has had an analogue world opened up to him or her as an infant. What emanates from this kind of development is a familiarity with the natural world and a gradual understanding and perception of its laws and operations. Human inventiveness and creativity incorporates the gradual development of transferrable skills throughout infancy, childhood and adolescence.

If the current collective understanding of what it means to be human is to be maintained, then an analogue based consciousness needs to be pre-eminent in any learning environment. The human body requires clear pathways in regard to both physical and psychological welfare to receive and interact efficiently with wavelength signals in an analogue world. The process of engaging digital technology and screen presence changes a child's perception of the body in regard to the space that it occupies. In an analogue environment the body moves within a vast natural world where life support involving bodily functions as well as sensual, emotional, physical and spiritual interaction occurs. Anticipation, hands-on interaction and memory create understanding, knowledge, perception and experience within breathable space. For the infant or child in this environment there is no requirement to measure or calculate. Digital and electronic media have substituted the notion of space as an environmental container of objects with that of space as something continually changing and extending beyond visual limits. This is a world created by measurement and number. The numbers create the illusion of space where perspective as a measurement device controls important aspects of perception. The mind has an intellectual part to play in this mindgame learning experience, however the body becomes almost redundant, other than acting as a life support for essential mental requirements to uphold the digital mantel. If children develop predominantly in this environment then the way they perceive their bodies is very different to that of the child who has been given the opportunity for many and varied analogue experiences.

Educational institutions need to look carefully at the introduction of digital learning to keep a balance between the experiences that enhance analogue based learning activity and the creation of digital opportunity. There would be a preference to engage students in Internet searches for art exhibitions rather than attending art galleries. Working with clay is messy and requires good organizational skills for students to have meaningful experiences with the material. In preference, a teacher may look at videos on the Internet with students about thumb pot creation or building with slabs. These videos are 
useful for instruction but only if accompanied by practical events. The use of interactive online games as an attempt to address truancy problems has some merit but surely should only be seen as a very short-term measure. Any perceived merit in an interactive online game or instruction should be based around prior experience in a natural or analogue environment. Even the creation of games associated with story telling should accompany an analogue understanding. After all, storytelling ought to be based around one's own experiences, not experiences predominantly gained through engaging in another person's constructed world. If the world of the $21^{\text {st }}$ Century requires the advancement in education of creativity and inventiveness, the curriculum needs to establish a balance between the analogue and the digital experiences of infants and children.

\section{Literature review}

Kathy Danko-McGhee and Rusian Slutsky (2007) state that being literate in the arts gives young children an advantage in learning to read and write. The overriding premise is that children best learn through multi sensory experiences. Language can no longer be viewed as being just oral and written. For children in any culture to develop as writers, they need time to kinesthetically experience scribbling, which builds the foundation to form lines associated with letter formation. Eisner (1975) talks about the benefits within the arts of creating, viewing and discussing. He states that the visual arts possess a broad spectrum of literacy skills that amplifies knowledge and understanding. Art based experiences expand vocabulary, comprehension and critical thinking. Arnheim (1974) states that we have neglected the gift of comprehending things through our senses and our eyes have been reduced to instruments with which to identify and measure. Kellogg (1970) is more specific in her research about infant and child development through drawing. She is more concerned about the universal aspects of children's art. The scribbles, circles and strokes drawn by a child combine to form the significant shape of a Mandala. Kellogg states that Mandalas are a key part of the sequence that leads from abstract work to pictorials. In exploring the world of perception, Merleau Ponty critiques the philosophical position that has held back the development of knowledge about vulnerable entities within society, including children. The position that he criticizes is the classical position that believes that the vocation of the human is to be "lord and master" of nature and to develop sovereign knowledge. Children are seen as "kept from the truth", a state of grace of which the fully-fledged human is capable. Merleau Ponty criticizes this position by inferring that it does not learn from the position that it criticizes. He states that in striving for sovereignty and coherence, the human actually falls well short and an understanding of the natural world is riddled with gaps, just like it is for the infant. However, the adult state of mind is a little more coherent than that of the infant mind.

\section{Contribution to Knowledge}

Creativity and inventiveness as a human process rely on the opportunity to develop natural and universal signs that point to an inner developmental process, often commensurate with personal capacity, as symbolic forms, scribbles, circles and strokes form images that represent a child's contribution to the community. Abstract shapes that become the basis of formal written language develop as a consequence, usually under the guidance of tutors and teachers. Numbers also form to enable the formal expression of concepts in numeracy. Each evolving symbol, made initially using strokes, then circles, will change as the child grows and finds more intricate ways to represent its world. When drawing, a child uses the visual elements of line, tone, texture etc. to record and express responses to experience. An imaginative world becomes chaptered through the expression of outward design and the subtleties of observation and play are thus externalized for communication purposes. Through experience, the child learns discernment, develops sensibilities, receives and evaluates responses and forms a sense of the aesthetic and the moral. During this time, the child is developing an imagination. The child is able to reorder an environment, developing imaginative worlds where it can live, and create identities that are outside its own limitations. A carefully planned but free early childhood needs to have well managed digital television watching. Everything is magical for a child. There exists a life beyond the visible world that can terrify children at times. Green monsters appear and at times seem to threaten an attack- a child waking up from a bad dream and vividly 
remembering the contents and wondering where it all went to. A child without a developed imagination through natural means as described could look at screen based technological offerings and wonder why scenes keep changing or why people and other objects appear and disappear so quickly, or look big and then suddenly can hardly be seen. With no real experience of personal power to interpret through drawing the child is left to react in some uncontrolled way or to be passive externally in a desire to please the parenting fraternity. This is, then, a dimension and experience for the child that is totally controlled by adults who have studied film technique and who are hired by organizations to create worlds for children worlds that sensationalize and speed up drastically some of life's processes that normally take time and allow for interaction to develop. In this regard, some look forward to the time when digital implants in the brain can also act as a catalyst for human development and negate the need for infant and childhood development. It is envisaged that a human would become a productive member of society much sooner and that this would also be a way to construct an ordered society!

Dreams are a source for the imagination over which the child must learn to have some power. The required power to control can be gained with the production of marks using the drawing and carving or cutting action. Broader sensual experiences of sound, touch, smell, taste as well as sight come into play and the jottings that occur can represent untapped sources for stories and further interaction with the environment. Privileged adults often tap into this phenomenon. Having been through the described analogue based experiences themselves, they write stories for children. This practice often fascinates participating children as it reinforces and legitimizes their experiences. It is interesting to note that authors often include adults as the intended audience. The source material for these stories is often forgotten or not experienced by adults in their early years and can only be experienced in an environment that is other than infantile. A light may be turned on in a person from some distant shore, but, alas, a systemic dependence can then become the basis for understanding, rather than a confidence in the developing self progressing in an environment that reflects a suitably naïve perception. In a similar way, many school students undertaking a "Photoshop" experience can quickly develop a fascination that involves the communal expression of the "cool" with the use of digital plug ins and effects creating a sea of clichés, rather than this medium becoming a way of intentionally expressing or formalizing ideas or concepts that come from an earlier time in the evolution of a body of work. The creation and expression of ideas and concepts is a difficult proposition for those who have little intuitive sense to undertake this process. In fact these students often unwittingly involve themselves in a learning environment that reflect infant and childhood approaches in an attempt to comply with an expected code of conduct. This kind of engagement by students often ceases or moves to other social domains for stylized expression. Voyages of discovery and mark-making become the essence of adventure. It is usually a minority of privileged students that are destined to succeed in an arts based learning environment. Encouragement to reorder environments occurs mainly for those select few who can both enter an institution and graduate in order to join the workforce in the production of this commercial or virtual world. Some human beings as students or as artists are able to create ideas and concepts and to deliver these through publication and public exhibition but a societal attitude to safe and conservative "common sense" appeal at times isolates artists from their public.

The rigors of strict pathways to employment schemes and the like eventually draw students away from fruitful sensual self-fulfillment that, in turn, is defined as an unproductive pursuit. In a social setting mainly driven by the production and sale of goods and services the human and societal aspirations of the individual are devalued. The ability to reorder environments and develop imaginary worlds and personal involvement in discernment and quality choice become limited as a result. Choice has a Pavlovian basis and digital technology does have the operational technique and power to seduce. The domain of chemical substances both natural and synthetic becomes an option for exploration and an escape from the heart-felt need for creative and spiritual personal fulfillment. Some measure of satisfaction can be gained from the packaged goods handed out in the form of templates in a commercial or virtual world but that underlying urge for fulfillment spoils a complete engagement in these digital gifts.

There is much potential for each citizen within each country (and globally) to be included and to take part in the digital environment, but meaningful participation in society requires a literate, creative and inventive involvement. The 
capacity for human advancement and temporal fulfillment for the individual, society and the planet is inherent in each individual. As has been mentioned earlier, the urge to be literate is inherent in the spark that comes with the gift of life. Societal systems need to value these early human phenomena very highly, with educational practice integrating with individual development in a seamless manner.

There is a real need to develop support mechanisms and systemic change to allow optimum conditions for infant and childhood growth that is not based around the requirements of older systems, largely built around the creation of wealth. These systems have been based around philosophies that in practice make no allowance for the patience required to attend to the needs of human individuality. Measuring how far infants, children and adolescents fall short of the requirements expected of an average, healthy person perceivably required in the workplace, is the product of a system seeking short term economic glorification. Such a system bases its educational processes around the practice of collecting together infants and children for the purpose of regimentation as a basic and overriding learning model at a time in their lives when children require the opposite scenario in order to develop as creative participators in a broader twenty first century economy. Regimentation of the collected and using the same rigid methods and number systems to control systemic failure and success is a process that has been based around an industrial model of education designed to produce workers whose deliberations do not rise above the level of the mundane. This situation is unfair on the individual seeking early opportunity as a global citizen and these methods no longer apply in a digital age or indeed a digitally based economy.

\section{Conclusion}

If a human being has a right to freedom, then each person has a right to have provided for them a rich analogue based environment within which to be nurtured and to grow. Infant and childhood development proceeds in a guided and unsteady way through trial and error. Progress is observable and at times spasmodic. As the child's sense receptors encounter analogue wavelengths, intricate details of sound, image, taste touch and smell bring unique knowledge to the individual who exercises an innate and learned capacity to respond. One response that an infant discovers is the ability to make marks.
Making sounds, touching and movement constitute other responses. Mark making becomes a progressive and a refining process as individual capacity strengthens, eventually allowing the drawing of shapes that emerge from initial scribbling. These shapes and symbols linked with other sensual exposure and brain activity become the basis of literacy requirements in society.

It must be stated here that there are many unanswered questions regarding the role that scribbling and drawing plays in the development of the infant. However, this universal human phenomenon, like breathing and other involuntary infant acts must have a function. In the case of scribbling and drawing, this function is linked to initial feelings of contact and control in the child's world. Scribbling and drawing is also linked to the development of a spatial awareness in the development and creation of symbols that, as has already been stated, are directly aligned to literacy and other forms of communication

Digital technology creates boundless opportunities for children who have been nurtured through exposure to analogue experiences. When suitably schooled, these children (more likely as slightly older human beings), can engage in a digitally based global system that does not place limits, such as mediocre expectation, on their growth within a digital environment. The constructive use of digital technology, therefore, requires children to have well-developed analogue sensibilities. Infant and child development that is predominantly based around screen and digital technology alone affects the developing human body differently to a body engaged in sorting out and responding to analogue stimuli. Digital technology requires bodily responses to be passive and for functions and reactions of the body to be a life support system that develops quick hands for mouse, keyboard and other such mechanical functions, as well as quick eyes for the screen. Therefore, the body is not as involved in kinetic movement and response, especially those actions in the natural environment that work together with intellectual pursuit to develop imagination, creativity and inventive pursuit in both the analogue and digital environments.

\section{References}

[1] Arnheim, Rudolph. Art and Visual Perception, Berkeley, CA: University of California, (1974). 
[2] Kellogg, Rhoda Analysing Children's Art, Mayfield Publishing Company, CA, (1970)

[3] Danko-McGhee, Cathy. Rusian, Slutsky. "The Impact of Early Art Experiences on Literacy Development.” National Art Education Association, VA, (2007).

[4] Eisner, Elliott, Educating Artistic Vision, Macmillan Publishing Company Co, New York: Collier, Macmillan Publishers, London, (1972).

[5] Merleau-Ponty, Maurice, The World of Perception, (2004). 